\title{
Inhomogeneous beam-like models for the dynamic analysis of multi- storey buildings
}

\author{
A. Greco, I. Fiore, S. Caddemi, I. Caliò \\ Department of Civil Engineering and Architecture, University of Catania, viale A. Doria 6, Catania, Italy
}

\section{G. Occhipinti}

Institute of Environmental Geology and Geoengineer, Italian National Research Council (CNR), Via Salaria $\mathrm{km}$ 29,300, Monterotondo, Rome (Italy)

\begin{abstract}
The present paper proposes a simplified inhomogeneous beam-like model, suitable for the dynamic analysis of buildings with non-uniform vertical distribution of mass and stiffness. Asymmetric distribution of stiffness within the floors, which leads to torsional effects, can be also taken into account. The equations of motion of the proposed spatial asymmetric beam-like model are derived through the application of Hamilton's principle. The linear dynamic behaviour of the inhomogeneous beam-like element is then evaluated by discretizing the continuous model according to a Rayleigh-Ritz approach based on a suitable number of modal shapes of uniform shear torsional beam. The comparison of the results with those obtained by means of three-dimensional FEM models shows the efficacy of the proposed approach.
\end{abstract}

\section{INTRODUCTION}

The advancements on computational procedures and parallel processing of the last few years enhance accurate dynamic analysis and seismic assessments of multi storey buildings. The analyses can be carried out at building or urban level and high fidelity models or sophisticated large scale simulations can be adopted respectively. In particular, the dynamic analysis of entire urban area needs optimized computation procedures and simplified but accurate numerical models. Thus, a new and renovated interest has grown recently on the beam-like models that have been introduced in the last century. The beamlike models, which are based on the equivalence of multi-level structures to flexural-shear coupling continuum beams, aim to simulate the dynamic behaviour of multi-level buildings by drastically reducing the computational effort. The simplified beam-like approaches firstly proposed in the literature make usually reference to homogeneous elements with uniform stiffness distribution (Chajes, et al., 1993). Some contributions take into account uniform beams with eccentricity between the centers of stiffness and mass (Zalka, 2014; Potzta \& Kollár, 2003; Piccardo, et al., 2019).

In the present paper an inhomogeneous beam-like model suitable for the schematization of buildings, which do not have uniform mass and stiffness distribution along the height and are characterized by asymmetric plans, is proposed. The floor deformability has been taken into account by means of floor stiffness reducing coefficients. The equations of motion of the proposed spatial asymmetric beam-like model are derived through the application of Hamilton's principle. The linear dynamic behaviour of the inhomogeneous beam-like element is then evaluated by discretizing the continuous model according to a Rayleigh-Ritz approach (Chopra, 2001). An appropriate number of modal shapes of a uniform shearonly continuum beam have been as sumed as shape functions in the discretization. Furthermore, the validation has been performed by comparing the results to the FEM models. The capabilities of the proposed model have been highlighted by evaluating the dynamic response of different multi-storey buildings and comparing the results to 3D FEM modelling approach.

\section{THE PROPOSED BEAM-LIKE MODEL}

In order to build a simplified model able to correctly reproduce the main dynamic characteristics of a multi-storey asymmetric building it is necessary to define the rules upon which the equivalence is based. In the present study the mass and stiffness of each inter-storey of the building, related to the presence of columns, shear walls and beams, is taken into account to provide the characteristics of the correspondent portion of the equivalent beam. As proposed by Piccardo et al. (Piccardo, et al., 2019) the equivalence is endorsed by means of the related deformation energy.

The characteristics of the continuum non uniform beam can be assumed according to one of the wellknown beam models already presented in literature. According to the aims of this research, the uniform shear torsional beam clamped at the base has been considered. This continuous model, as it will be shown, is the simplest but at the same time reliable, 
beam-like model for the analysis of the dynamic behaviour of low- and mid-rise buildings.
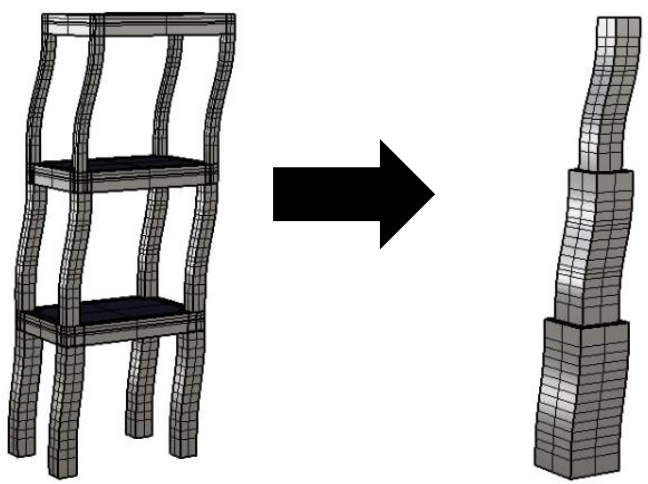

Figure 1. The beam-like model

A fundamental hypothesis, which strongly influences some of the studies proposed in the literature, is related to the presence of rigid floors.

According to this simplification, the rigid out of plane behaviour of each floor affects all the columns ends at the floor level. Consequently, all the columns ends rotations coincide with the rigid floor rotation.

The evaluation of the shear stiffness of the equivalent beam is performed similarly to what proposed in (Piccardo, et al., 2019) and is based on the building's geometric and elastic properties.

The equivalent beam shear stiffnesses $\left(S_{x}, S_{y}\right)$ - in the two principal directions - and torsional $(C)$ stiffness are obtained for each inter-storey by considering the contributes of all the $N$ resistant elements of coordinates $x, y$ (for instance columns or shear walls) as Equation 1 shows.

$$
\begin{aligned}
& S_{x}=k_{x} \sum_{i=1}^{N} S_{x i} \\
& S_{y}=k_{y} \sum_{i=1}^{N} S_{y i} \\
& C=k_{c}\left(\sum_{i=1}^{N} C_{i}+\sum_{i=1}^{N} S_{x i} y_{i}^{2}+\sum_{i=1}^{N} S_{y i} x_{i}^{2}\right)
\end{aligned}
$$

Correction coefficients $k_{x}, k_{y}, k_{c}$ have been introduced in Equation 1 (in the case of rigid floor $k_{x}=k_{y}$ $\left.=k_{c}=1\right)$ in order to take into account the floor deformability.

The mentioned coefficients have been calculated by means of an optimization procedure based on the minimization of an opportune objective function, reported in Equation 2. The latter measures the differences between the first three frequencies of vibration calculated by means of the beam like-model $\left(\omega^{\text {calc }}\right)$ and the correspondent available ones $\left(\omega^{\text {target }}\right)$, obtained either through FEM models or measured on the buildings.
$O\left(\omega_{j}\right)=\sqrt{\sum_{j=1}^{3}\left(\frac{\omega_{j}^{\text {calc }}-\omega_{j}^{\text {target }}}{\omega_{j}^{\text {target }}}\right)^{2}}$

The minimization procedure seeks iteratively the set of correction coefficients which give the best correspondence with the target frequencies of the building.

The influence of the floor out of plane stiffness on the dynamic characteristics of the beam-like model is discussed in the applicative section of the present paper.

\subsection{Dynamic behavior of the asymmetric beam-like model}

With reference to the $k$-th storey of the building in the X-Y plane, shown in Figure 2, the centers of mass and stiffness will be assumed not coincident to each other (due for example to the presence of shear walls reported in black in the figure) thus inducing a torsional behaviour of the building.

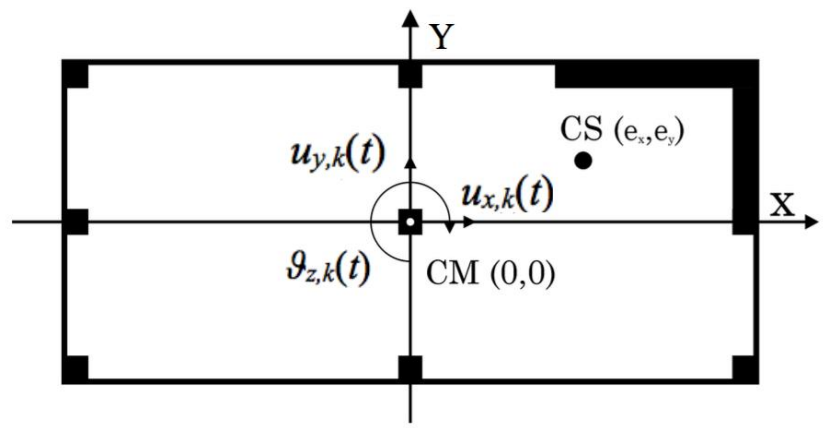

Figure 2. Example of asymmetric storey (with eccentricity between center of mass CM and center of stiffness CS) and its displacement components in the plane $\mathrm{X}-\mathrm{Y}$

Figure 2 reports the $k$-th storey and the displacements $u_{x, k}(t), u_{y, k}(t)$ and rotation $\vartheta_{z, k}(t)$ of the center of mass CM. The center of stiffness CS of coordinates $\left(e_{x}, e_{y}\right)$ is also indicated .

The masses $M_{x k}$ and $M_{y k}$ of the $k$-th storey and its second order moment $I_{o k}$ are assumed to be concentrated at the floor levels, while the correspondent values $m_{x}(z) m_{y}(z)$ and $I_{o}(z)$ are assumed to be constant within each inter-storey length of the equivalent beam (but different along the height). Displacements and rotations of the beam are also assumed as continuous values $u_{x}(z, t) u_{y}(z, t), \vartheta_{z}(z, t)$. Seismic excitations denoted by displacements $u_{g x}(t)$, $u_{g y}(t)$ at the base $z=0$ are considered in the general formulation. In the following equations, primes and dots denote differentiation with respect to position $z$ and time $t$, respectively.

The kinetic energy, related to total displacements, is therefore given by Equation 3. 
$T=\left(\frac{1}{2} \int_{0}^{h} m_{x}(z)\left[\dot{u}_{x}(z, t)+\dot{u}_{g x}(t)\right]^{2} d z+\right.$

$+\frac{1}{2} \int_{0}^{h} m_{y}(z)\left[\dot{u}_{y}(z, t)+\dot{u}_{g y}(t)\right]^{2} d z+$

$+\frac{1}{2} \int_{0}^{h} I_{o}(z)\left[\dot{\vartheta}_{z}(z, t)\right]^{2} d z+$

$+\frac{1}{2} \sum_{k=1}^{N} M_{x k}\left[\dot{u}_{x, k}(t)+\dot{u}_{g x}(t)\right]^{2}+$

$\left.\frac{1}{2} \sum_{k=1}^{N} M_{y k}\left[\dot{u}_{y, k}(t)+\dot{u}_{g y}(t)\right]^{2}+\frac{1}{2} \sum_{k=1}^{N} I_{o k}\left[\dot{\vartheta}_{z, k}(t)\right]^{2}\right)$

Consistently with the adopted beam model, the elastic energy is associated with the shear deformation of the beam and its torsional stiffness. This result is therefore given by Equation 4 .

$U=\left(\frac{1}{2} \int_{0}^{h} G A_{x}(z)\left[u_{x}^{\prime}(z, t)-e_{y} \vartheta_{z}^{\prime}(z, t)\right]^{2} d z+\right.$

$+\frac{1}{2} \int_{0}^{h} G A_{y}(z)\left[u_{y}^{\prime}(z, t)+e_{x} \vartheta_{z}^{\prime}(z, t)\right]^{2} d z+$

$\left.+\frac{1}{2} \int_{0}^{h} G J_{z}(z)\left[\vartheta_{z}^{\prime}(z, t)\right]^{2} d z\right)$

where $G A_{x}, G A_{y}$ are the shear stiffnesses in the $x$ and $y$ directions, respectively, and $G J_{z}$ the torsional stiffness of the beam (Equation 5).

$$
\begin{aligned}
& G A_{x}=S_{x} \cdot h \\
& G A_{y}=S_{y} \cdot h \\
& G J_{z}=C \cdot h
\end{aligned}
$$

The application of Hamilton's principle in the absence of non-conservative forces allows to obtain the equations of the motion of the continuous beam having infinite degrees of freedom that are not reported for the sake of brevity.

In order to evaluate the response of the equivalent continuous beam by introducing a limited number of degrees of freedom, a Rayleigh-Ritz discretization has been performed. The latter is based on the use of $n$ modal shapes of the homogeneous shear beam of total length $h$ clamped at the base having equations:

$$
\psi_{m}(\zeta)=\sin \left(\frac{\pi}{2}(2 m-1) \zeta\right) \quad m=1,2, \ldots, \infty
$$

where $\zeta=z / h$ is the dimensionless abscissa of the beam.

All the introduced displacements can therefore be expressed as shown in Equations 7 and 8.

$$
\begin{aligned}
& u_{x}(\zeta, t)=\sum_{i=1}^{n} \psi_{i}(\zeta) q_{i_{x}}(t)=\sum_{i=1}^{n} \psi_{i} q_{i_{x}} \\
& u_{y}(\zeta, t)=\sum_{i=1}^{n} \psi_{i}(\zeta) q_{i_{y}}(t)=\sum_{i=1}^{n} \psi_{i} q_{i_{y}} \\
& \vartheta_{z}(\zeta, t)=\sum_{i=1}^{n} \psi_{i}(\zeta) q_{i_{g}}(t)=\sum_{i=1}^{n} \psi_{i} q_{i_{g}} \\
& u_{x, k}(t)=\sum_{i=1}^{n} \psi_{i, k} q_{i_{x}}(t)=\sum_{i=1}^{n} \psi_{i, k} q_{i_{x}} \\
& u_{y, k}(t)=\sum_{i=1}^{n} \psi_{i, k} q_{i_{y}}(t)=\sum_{i=1}^{n} \psi_{i, k} q_{i_{y}} \\
& \vartheta_{z, k}(t)=\sum_{i=1}^{n} \psi_{i, k} q_{i_{g}}(t)=\sum_{i=1}^{n} \psi_{i, k} q_{i_{g}}
\end{aligned}
$$

Being $q$ the generalized coordinates, which represent the contribution of the single shape function to the total response, and $\psi_{i, k}$ are the values of $\psi_{i}(\zeta)$ calculated at level $k$.

The equations of motion in the generalized space ass ume therefore the following form:

$\mathbf{M}^{*} \ddot{\mathbf{q}}+\mathbf{K}^{*} \mathbf{q}=\mathbf{P}^{*}$

where the generalized mass and stiffness matrices can be expressed as:

$$
\begin{aligned}
\mathbf{M}^{*} & =\left[\begin{array}{lll}
\mathbf{M}_{x}^{*} & & \\
& \mathbf{M}_{y}^{*} & \\
& & \mathbf{M}_{\vartheta}^{*}
\end{array}\right] \\
\mathbf{K}^{*} & =\left[\begin{array}{lll}
\mathbf{K}_{x}^{*} & & \mathbf{K}_{x \vartheta}^{*} \\
& \mathbf{K}_{y}^{*} & \mathbf{K}_{y \vartheta}^{*} \\
\mathbf{K}_{\vartheta_{x}}^{*} & \mathbf{K}_{\vartheta_{y}}^{*} & \mathbf{K}_{\vartheta}^{*}
\end{array}\right]
\end{aligned}
$$

In detail, each element of the previous matrices is reported in Equations 12 and 13.

$$
\begin{aligned}
& \mathbf{K}_{i j_{x}}^{*}=\frac{1}{h} \int_{0}^{1} G A_{x} \psi_{i}^{\prime} \psi_{j}^{\prime} d \zeta \\
& \mathbf{K}_{i j_{y}}^{*}=\frac{1}{h} \int_{0}^{1} G A_{y} \psi_{i}^{\prime} \psi^{\prime}{ }_{j} d \zeta \\
& \mathbf{K}_{i j_{\vartheta}}{ }^{*}=\frac{1}{h} \int_{0}^{1}\left(G J_{z}+e_{y}^{2} G A_{x}+e_{x}^{2} G A_{y}\right) \psi^{\prime}{ }_{i} \psi^{\prime}{ }_{j} d \zeta \\
& \mathbf{K}_{i j_{x \vartheta}}^{*}=\mathbf{K}_{i j_{9 x}}^{*}=-\frac{1}{h} \int_{0}^{1} e_{y} G A_{x} \psi^{\prime}{ }_{i} \psi^{\prime}{ }_{j} d \zeta \\
& \mathbf{K}_{i j_{y,}}{ }^{*}=\mathbf{K}_{i j_{y y}}{ }^{*}=\frac{1}{h} \int_{0}^{1} e_{x} G A_{y} \psi^{\prime}{ }_{i} \psi^{\prime}{ }_{j} d \zeta
\end{aligned}
$$


$\mathbf{M}_{i j_{x}}^{*}=h \int_{0}^{1} m_{x} \psi_{i} \psi_{j} d \zeta+\sum_{k=1}^{N} M_{x, k} \psi_{i, k} \psi_{j, k}$

$\mathbf{M}_{i j_{y}}^{*}=h \int_{0}^{1} m_{y} \psi_{i} \psi_{j} d \zeta+\sum_{k=1}^{N} M_{y, k} \psi_{i, k} \psi_{j, k}$

$\mathbf{M}_{i j_{g}}^{*}=h \int_{0}^{1} I_{z} \psi_{i} \psi_{j} d \zeta+\sum_{k=1}^{N} I_{o, k} \psi_{i, k} \psi_{j, k}$

The presence of a ground motion provides the following generalized load vector:

$\mathbf{P}_{i j}^{*}=\left[\begin{array}{l}\mathbf{P}_{x}^{*} \\ \mathbf{P}_{y}^{*} \\ \mathbf{P}_{\vartheta}^{*}\end{array}\right]$

whose components are :

$\mathbf{P}_{j_{x}}^{*}=-\ddot{u}_{g x} h \int_{0}^{1} m_{x} \psi_{j} d \zeta-\ddot{u}_{g x} \sum_{k=1}^{N} M_{x, k} \psi_{j, k}$

$\mathbf{P}_{j_{y}}^{*}=-\ddot{u}_{g y} h \int_{0}^{1} m_{y} \psi_{j} d \zeta-\ddot{u}_{g y} \sum_{k=1}^{N} M_{y, k} \psi_{j, k}$

$\mathbf{P}_{\vartheta}^{*}=0$

The modes of vibration $\mathbf{q}$ in the generalized space and the vibration frequencies $\omega$ of the equivalent beam are obtained from the resolution of the following eigen-problem.

$$
\left[\mathbf{K}^{*}-\omega^{2} \mathbf{M}^{*}\right] \mathbf{q}=\mathbf{0}
$$

Equations (7-8) allow to express the modal shapes of the equivalent beam in the geometric space.

\section{APPLICATIONS}

In this section the proposed procedure has been applied to two benchmarks. For each of them the modes and the frequencies of vibration provided by analyzing the shear torsional equivalent beam have been compared to those obtained through a finite element model of the building implemented in SAP2000 (CSI, 2007).

The two benchmarks aim to simulate, in a simplified way, buildings with real inhomogeneous geometric properties. Due to that, vertical stiffness variation and eccentricity of the center of stiffness are considered, respectively.

\subsection{Benchmark 1: building with inhomogeneous vertical stiffness}

The first benchmark is a six-storey building with 9 columns whose symmetric plan is reported in Figure 3.

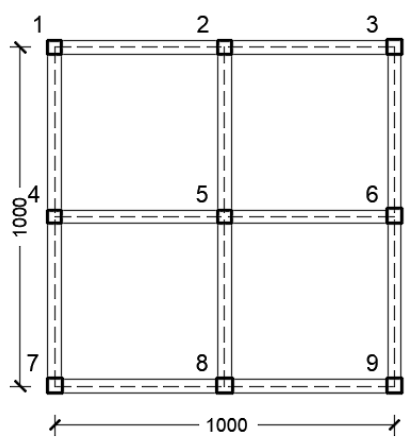

Figure 3. Bench mark 1 - plan

The building has a non-uniform vertical distribution of mass and stiffness since the size of the cross sections of all the columns is assumed to be decreasing along the height of the building. This condition usually affects several existing buildings built before the modern seismic codes (G.Occhipinti, et al, 2017; G.Occhipinti, et al, 2018). In detail, the following cross sections have been considered: $45 \times 45$ $\mathrm{cm}^{2}$ at the first and second elevation; $40 \times 40 \mathrm{~cm}^{2}$ at the third floor; $35 \times 35 \mathrm{~cm}^{2}$ at the fourth level; $30 \times 30$ $\mathrm{cm}^{2}$ at the last two floors.

The cross sections of all the beams is assumed to be $30 \times 50 \mathrm{~cm}^{2}$. The inter-storey height is $3 \mathrm{~m}$ and the columns have been assumed clamped at the base. The floor thickness is $25 \mathrm{~cm}$ at each level. The constituent material is assumed to be elastic and characterized by Young's modulus of $31250 \mathrm{MPa}$, a Poisson ratio equal to 0.2 and a specific weight of 25 $\mathrm{kN} / \mathrm{m}^{3}$.

For the evaluation of the dynamic characteristics of the building and of the equivalent beam only the self-weight of the members have been considered with no extra loading.

The first 6 frequencies of vibration of the proposed beam-like model are reported in Table 1 both in the hypothesis of rigid (BL-RF) and deformable (BL) floor and compared to the results obtained through a finite element discretization of the building (FEM).

As it can be observed the obtained results show a good agreement especially when the floor deformability is taken into account by means of the correction coefficients already discussed in section 2 and introduced in Equation 1.

Also the correspondent modes of vibration have been plotted and compared, showing a good agreement. For the sake of brevity only the first three modes are shown in Figure 4.

The best values of the stiffness correction coefficients, identified by means of the minimization of the objective function proposed in Equation 2, are in this case: $k_{x}=k_{y}=0.6$ and $k_{c}=0.701$. 
Table 1. Comparison of the modal periods.

\begin{tabular}{|c|c|c|c|}
\hline \multirow[t]{2}{*}{ Mode } & BL-RF & $\overline{B L}$ & $\overline{F E M}$ \\
\hline & $\mathrm{S}$ & $\mathrm{S}$ & $\mathrm{S}$ \\
\hline 1 & 0.504 & 0.650 & 0.653 \\
\hline 2 & 0.504 & 0.650 & 0.653 \\
\hline 3 & 0.409 & 0.489 & 0.483 \\
\hline 4 & 0.202 & 0.261 & 0.257 \\
\hline 5 & 0.202 & 0.261 & 0.257 \\
\hline 6 & 0.167 & 0.200 & 0.200 \\
\hline
\end{tabular}

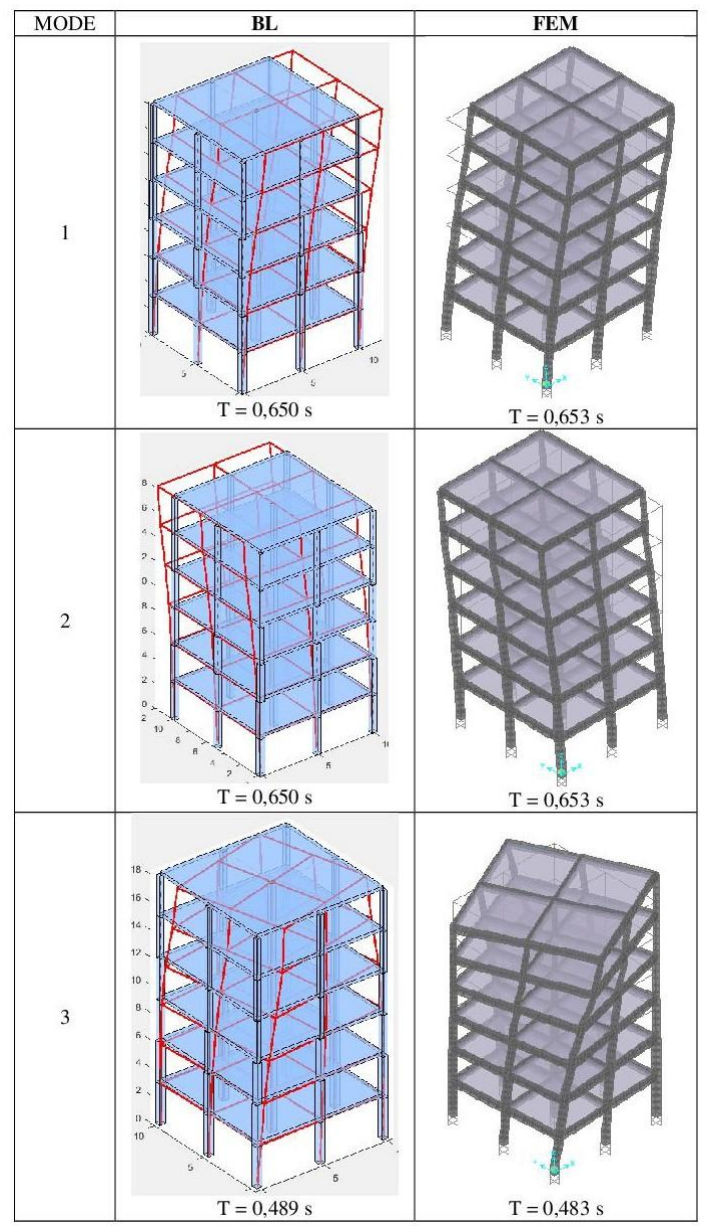

Figure 4. The first three modes of vibration for case study 1 obtained through the beam-like model (BL) and the FEM discretization

\subsection{Benchmark 2: buildings with planar asymmetry}

As a second case study, a parametric analysis has been performed on a building with the irregular plan shown in Figure 5. This benchmark is a simplified representation of eccentricity in concrete frames, commonly noticed in existing buildings. In case of irregular plan, the eccentricity may be extremely considerable and negatively affect the seismic response of the structure.

The cross section size of the columns denoted as $1,2,4$ and 5 is assumed to be $40 \times 40 \mathrm{~cm}^{2}$ while the for the other columns dimensions $45 \times 45 \mathrm{~cm}^{2}$ are employed.

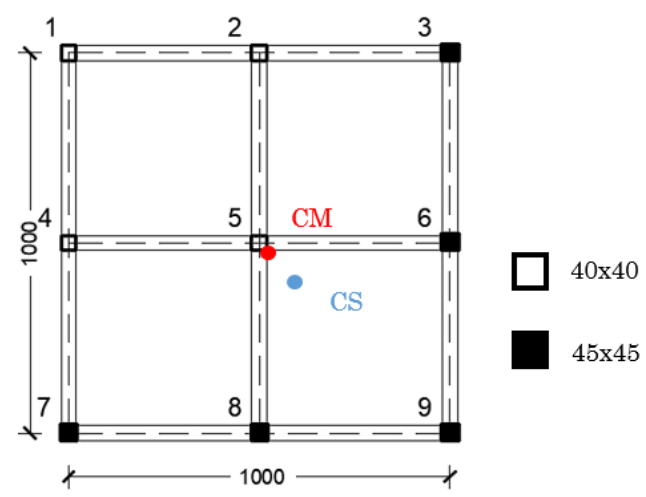

Figure 5. Bench mark 2 - plan

Due to this unsymmetrical arrangement of the columns an eccentricity between the centers of stiffness (CS) and mass (CM) can be noticed, as the Figure 5 shows.

A parametric analysis on the periods of vibration has been carried out on buildings with number of floors varying from 4 to 16 . As already done in the previous section, the beam like model with rigid (BL-RF) and deformable (BL) floors have been analyzed and compared to those obtained by means of SAP2000 (FEM).

Figure 6 shows the variation of the first three periods of vibration according to the number of storeys. A good agreement between the results obtained by means of the beam like model with deformable floors (BL) and the finite element discretization (FEM) has been obtained. Although a good correspondence has also been observed for higher periods of vibration the results have not been reported in the paper for brevity.

Even though the vibration periods are underestimated in Modes 1 and 2 (Figure 6.a and 6.b) and over-estimated in Mode 3 (Figure 6.c) the difference is always less than $10 \%$. The latter approximation is considered acceptable in view of the adopted simplified model.

The results highlight the efficacy of the proposed procedure and the importance of the correction coefficients proposed in this study.

The values of the correction coefficients $k_{x}, k_{y}, k_{c}$ introduced in Equation 1 have been obtained solving an optimization problem for each building in the present parametric analysis.

Figure 7 shows the variation of the coefficients in terms of number of floors. As it can be observed, the minimization of the objective function reported in Equation 2 provides an unique value (equal to 0.6 ) for the correction coefficients affecting the interstorey shear stiffness in the two directions independently on the number of floors of the building. On the other hand, the torsional correction coefficient $k_{c}$ initially decreases with the number of storey reaching the constant value of 0.6 for buildings of 8 floors or more. 

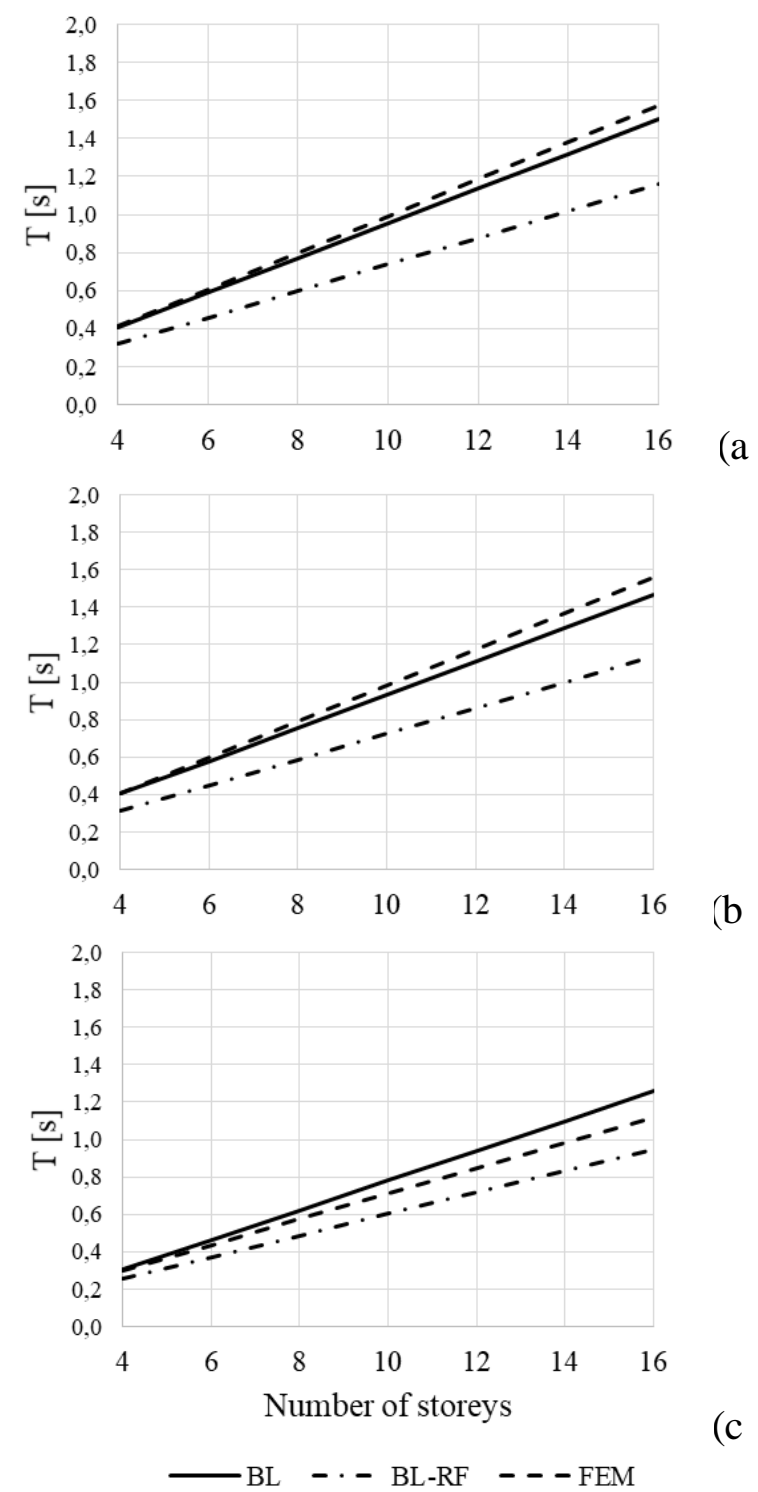

Figure 6. Variation of vibration periods with the number of floors in Mode1 (a); Mode 2 (b) and Mode 3 (c).

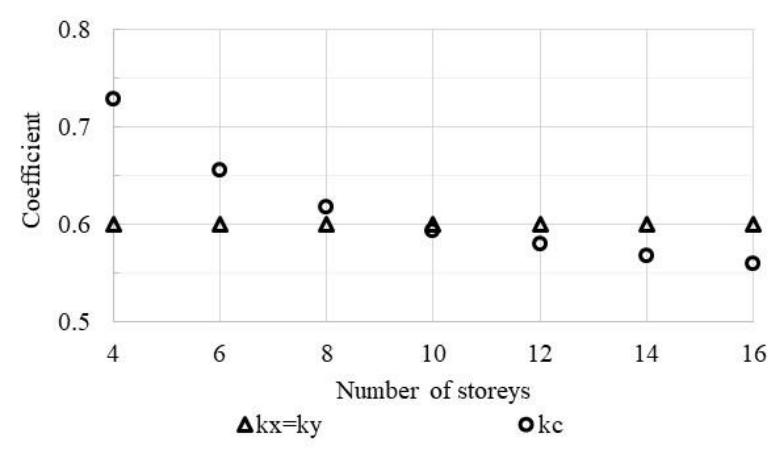

Figure 7. Variation of the correction coefficients with the number of floors.

\section{CONCLUSIONS}

The study proposes an inhomogeneous beam-like model suitable for the schematization of buildings, which do not have uniform mass and stiffness distribution along the height and are characterized by asymmetric plans. Appropriate stiffness correction coefficients have been introduced in order to take into account the floor deformability.

The linear dynamic behaviour of the inhomogeneous beam-like element is evaluated by discretizing the continuous model according to a Rayleigh-Ritz approach based on a suitable number of modal shapes of the uniform shear torsional beam clamped at the base. In spite of the simplicity of the proposed beam like model, very accurate results have been obtained. In particular frequencies and modes of vibration of different multi-storey buildings, evaluated by means of 3D FEM modelling approach, have been compared to the correspondent results for the beamlike models showing a very good correspondence.

The examples shown in the application section analyze separately the effects of inhomogeneous vertical stiffness distribution and planar asymmetry that often characterize existing build ings. However it has to be remarked that the proposed model is able to take into account contemporarily both the above mentioned irregularities.

\section{ACKNOWLEDGEMENT}

The authors acknowledge the financial support of the Ministero dell'Istruzione, dell'Università e della Ricerca (National Research Project PRIN 2015JW9NJT “Advanced mechanical modeling of new materials and structures for the solution of 2020 Horizon challenges").

\section{REFERENCES}

Chajes, M., Romstad, K. \& McCallen, D., 1993. Analysis of multiple-bay frames using continuum model. Journal of Structural Engineering ASCE, 119(2), pp. 522-546.

Chopra, A. K., 2001. Dynamics of Structures, Theory and Application to Earthquake Engineering. In: Dynamics of Structures, Theory and Application to Earthquake Engineering. Upper Saddle River: Prentice Hall.

CSI, 2007. CSI Analysis Reference Manual for SAP2000. s.1.:Computers and Structures Inc..

G.Occhipinti, B.Izzuddin, L.Macorini \& I.Ca liò, 2017. Realistic 3D Nonlinear Dynamic Analysis Of Existing And Retrofitted Multi-Storey RC Buildings Subject To Earthquake Loading. Rhodes Island, Greece, s.n.

G.Occhipinti, B.Izzuddin, L.Macorini \& I.Caliò, 2018. Realistic Seismic Assessment Of RC Buildings With Masonry Infills Using 3D High-Fidelity Simulations. Glasgow, s.n.

Piccardo, G., Tubino, F. \& Luongo, A., 2019. Equivalent Timoshenko linear beam model for the static and dynamic analysis of tower buildings. Applied Mathematical Modelling, Volume 71, pp. 77-95.

Potzta, G. \& Kollár, L. P., 2003. Analysis of building structures by replacement sandwich beams. Int. J. Solids Struct., 40(3), pp. 535-553.

Zalka, K., 2014. Maximum deflection of asymmetric wallframe buildings under horizontal load. Periodica Polytechnica: Civil Engineering, 58(4), pp. 387-396. 